\title{
POSSIBILITY OF CONVECTION FOR DIFFUSION (NEWTONIAN) VISCOSITY IN THE ICE SHELL OF EUROPA?
}

\author{
VALLE LÓPEZ \\ Seminar on Planetary Sciences, Facultad de Ciencias Geológicas, Universidad Complutense de \\ Madrid, 28040 Madrid, Spain \\ ROSA TEJERO and JAVIER RUIZ \\ Departamento de Geodinámica, Facultad de Ciencias Geológicas, Universidad Complutense de \\ Madrid, 28040 Madrid, Spain (E-mail: jaruiz@geo.ucm.es)
}

(Received 6 July 2004; Accepted 7 February 2005)

\begin{abstract}
Recent investigations into convection in Europa's ice shell have been based on nonNewtonian (stress-dependent) or Newtonian (stress-independent) viscosity for water ice. However, despite the wide use of Newtonian convection, experimentally observed water ice flow is non-Newtonian, and analysis of stability against convection of the ice shell using updated flow laws has been only performed for non-Newtonian rheologies. Here we use the flow law proposed for diffusion creep to analyze the possibility of the onset of convection for Newtonian viscosity in relation to the thermal state of Europa. Our findings indicate that for diffusion creep convection might have started, but that significantly lower heat flows (and equivalently higher shell thicknesses) and/or grain sizes are required than for superplastic flow, which is the most probable flow mechanism if the ice shell is convective.
\end{abstract}

Keywords: Convection, Europa, heat flow, rheology, thermal evolution

\section{Introduction}

The possibility, style, or consequences of solid-state convection in the ice shell of Europa have been the focus of several recent investigations. An important source of uncertainty in these studies is the rheology of water ice. The flow of water ice I, as observed in experimental conditions, is in fact non-Newtonian (i.e., stress-dependent, e.g., Durham and Stern, 2001; Goldsby and Kohlstedt, 2001). According to these experimental results, it has been proposed that, in planetary conditions, the dominant water ice flow mechanism should be superplastic flow, in which grain boundary sliding is the main process (Goldsby and Kohlstedt, 2001). Thus, superplastic flow (or grain size sensitive creep, as named by Durham and Stern, 2001) has been used in several studies of convection on Europa (Pappalardo et al., 1998; McKinnon, 1999; Nimmo and Manga, 2002; Barr and Pappalardo, 2003; Ruiz and Tejero, 2003). 
Nevertheless, other recent studies of Europa (Hussmann et al., 2002; Nimmo and Manga, 2002; Sotin et al., 2002; Spohn and Schubert, 2003), including numerical investigations (Tobie et al., 2003; Showman and Han, 2004), have used Newtonian (stress-independent) viscosity for water ice. However, the stability against convection of the shell has been not previously analyzed for realistic Newtonian viscosities, and diffusion (Newtonian) creep has not been experimentally observed in water ice (although a flow law for diffusion creep has been proposed from the values of relevant constants; Goldsby and Kohlstedt, 2001). Moreover, stress-dependent and stress-independent convection are different. Preliminary numerical simulations of superplastic flow convection on Europa (Barr and Pappalardo, 2003) find different styles (lower interior temperatures and a thinner stagnant lid) and potential geological implications (thinner brittle and elastic lithospheres) than would be the case for Newtonian convection.

There is therefore a need to specifically evaluate the possibility of convection on Europa's ice shell for a realistic Newtonian rheology. To this end, we used the flow law proposed for diffusion creep to analyze the possibility of the onset of convection in the ice shell in relation to the thermal state of Europa, and then we compare our results with those previously obtained for superplastic flow and dislocation creep (McKinnon, 1999; Ruiz and Tejero, 2003).

\section{Methodology}

As tidal heating in Europa's ice shell is strongly temperature-dependent (Ojakangas and Stevenson, 1989), the part of a conductive shell that contributes most to total heat flow is the deep and warm ice near its base. Accordingly, it may be considered that the conductive shell is heated from below. The stability against convection of a conductive layer heated from below is usually described by means of the Rayleigh number, which can be defined at the base layer viscosity,

$$
R a_{\mathrm{b}}=\frac{\alpha \rho g h^{3} \Delta T}{\kappa \eta_{\mathrm{b}}},
$$

where $\alpha$ is the thermal expansion coefficient, $\rho$ is the density $\left(930 \mathrm{~kg} \mathrm{~m}^{-3}\right.$ for water ice), $g$ is the acceleration due to gravity, $h$ is the layer thickness, $\Delta T=T_{\mathrm{b}}-T_{\mathrm{s}}$ is the temperature difference between the base and the surface of the layer, $\kappa$ is the thermal diffusion coefficient, and $\eta_{\mathrm{b}}$ is the effective viscosity at the layer base. For Europa, $g=1.31 \mathrm{~m} \mathrm{~s}^{-2}$, and $T_{\mathrm{s}}=100 \mathrm{~K}$. For water ice $\alpha=\alpha_{\mathrm{o}} T$ and $\kappa=\kappa_{\mathrm{o}} / T^{2}$, where $\alpha_{\mathrm{o}}=6.24 \times 10^{-7} \mathrm{~K}^{-2}$, and 
$\kappa_{\mathrm{o}}=9.1875 \times 10^{-2} \mathrm{~m}^{2} \mathrm{~K}^{2} \mathrm{~s}^{-2}$ (Kirk and Stevenson, 1987); both functions are calculated for $T_{\mathrm{i}}$, the temperature of the well-mixed interior in the case of convection. from

The viscosity for diffusion creep can be calculated in terms of temperature

$$
\eta=\frac{d^{2}}{2 A} \exp \left(\frac{Q}{R T}\right)
$$

where $d$ is the grain size, $Q$ is the activation energy for creep deformation, and $R=8.3145 \mathrm{~J} \mathrm{~mol}^{-1} \mathrm{~K}^{-1}$ is the gas constant. For grain sizes much larger than the grain boundary width, $A=42 V_{\mathrm{m}} D_{\mathrm{ov}} / R T_{\mathrm{i}}$ (Goldsby and Kohlstedt, 2001; Nimmo and Manga, 2002), where $V_{\mathrm{m}}$ is the molar volume and $D_{\mathrm{ov}}$ is the volume diffusion pre-exponential coefficient. The constant values experimentally obtained (Goldsby and Kohlstedt, 2001, and references therein) are $Q=59.4 \mathrm{~kJ} \mathrm{~mol}^{-1}, V_{\mathrm{m}}=1.97 \times 10^{-5} \mathrm{~m}^{3}$, and $D_{\text {ov }}=9.1 \times 10^{-4} \mathrm{~m}^{2} \mathrm{~s}^{-1}$.

Water ice has a strongly temperature-dependent thermal conductivity, given by $k=k_{\mathrm{o}} / T$, where $k_{\mathrm{o}}=567 \mathrm{~W} \mathrm{~m}^{-1}$ (Klinger, 1980). For this reason, $h$ is not the real thickness of the shell, but it is an effective thickness (McKinnon, 1999) that corresponds to a thermal equilibrium situation with a constant $k$ calculated for $T_{\mathrm{i}}$,

$$
h=\frac{k_{\mathrm{o}} \Delta T}{F T_{\mathrm{i}}}=\frac{b \Delta T}{T_{\mathrm{i}} \ln \left(T_{\mathrm{b}} / T_{\mathrm{s}}\right)},
$$

where $F$ is the vertical heat flow through the shell, and $b$ is the real ice shell thickness corresponding to $F$. $T_{\mathrm{b}}$ obviously corresponds to the melting point of water ice, which is a function of pressure (Chizhov, 1993): $T_{\mathrm{b}}=273.16$ $(1-p / 395.2 \mathrm{MPa})^{1 / 9}$, where $p=\rho \mathrm{g} b$.

Under the conditions of icy satellites convection would occur in the stagnant lid regime (due to the large viscosity variations across an ice shell), in which there is an immobile lid over the convective sublayer. In this case, the critical value of $R a_{\mathrm{b}}$ for the onset of convection is given by (e.g., Solomatov, 1995)

$$
R a_{\mathrm{b}}^{*}=20.9\left(\frac{Q \Delta T}{R T_{\mathrm{i}}^{2}}\right)^{4}
$$

and $T_{\mathrm{i}}$ can be approached according to (Ruiz and Tejero, 2003) 


$$
T_{\mathrm{i}} \approx\left(\frac{Q^{2}}{4 R^{2}}+\frac{Q T_{\mathrm{b}}}{R}\right)^{1 / 2}-\frac{Q}{2 R}
$$

Although $R a_{\mathrm{b}}{ }^{*}$ is nearly constant over the range of values analyzed, it is explicitly calculated in terms of $F$ (and, consequently, of $b$ ).

Diffusion creep is a grain-size-sensitive flow mechanism: grain sizes of 0.1 , 0.3 and $1 \mathrm{~mm}$ were used in the calculations. Spectral analysis of young fractures in the Tyre region suggests that the mean grain size of the shallow subsurface layer is $0.1 \mathrm{~mm}$ or greater (Geissler et al., 1998), and tidal straining would imply grain sizes not greater than $\sim 1 \mathrm{~mm}$ (McKinnon, 1999).

\section{Results and Conclusions}

Our results in Figures 1 and 2 show $R a_{\mathrm{b}}{ }^{*}$ and $R a_{\mathrm{b}}$ in terms of heat flow and ice shell thickness. For the purpose of this work, results are most significant when interpreted in terms of the heat flow required for the onset of convection (Figure 1). Since $R a_{\mathrm{b}}$ increases with cooling of the conductive shell (due to the increasing ice shell thickness), the onset of convection requires that the heat flow be lower than a critical value. This allows us to relate the feasibility analysis for convection initiation to the thermal state of Europa. A higher effective surface temperature (caused, for example, by an insulating regolith layer (e.g., Shoemaker et al., 1982; Ross and Schubert, 1987), or by a

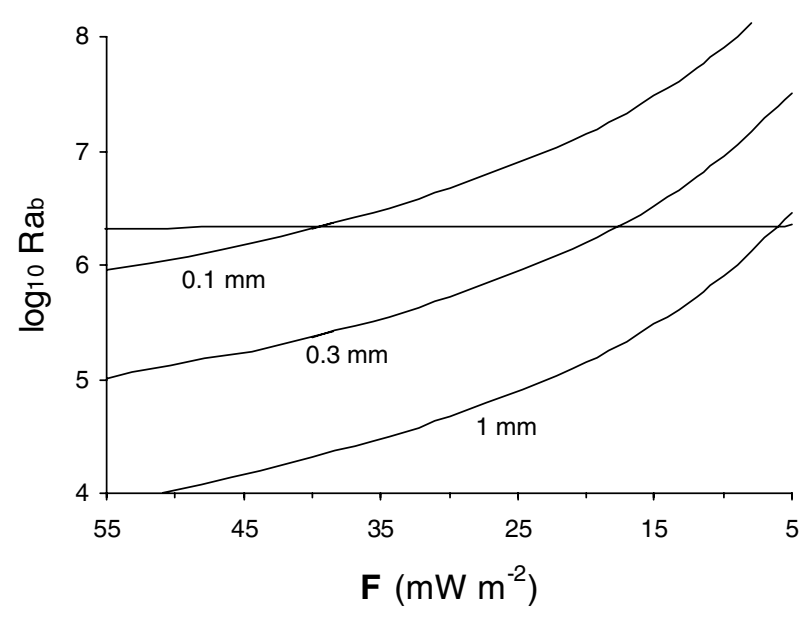

Figure 1. $R a_{\mathrm{b}}$ (labeled curves) and $R a_{\mathrm{b}} *$ (subhorizontal line) in terms of the heat flow through a conductive ice shell on Europa, $F$, for diffusion creep, $T_{\mathrm{s}}=100 \mathrm{~K}$, and grain sizes of $0.1,0.3$, $1 \mathrm{~mm}$. When $R a_{\mathrm{b}}>R a_{\mathrm{b}}{ }^{*}$, the shell is unstable and solid-state convection is initiated. Heat flow is represented in reverse order to show how $R a_{\mathrm{b}}$ increases with shell cooling. 


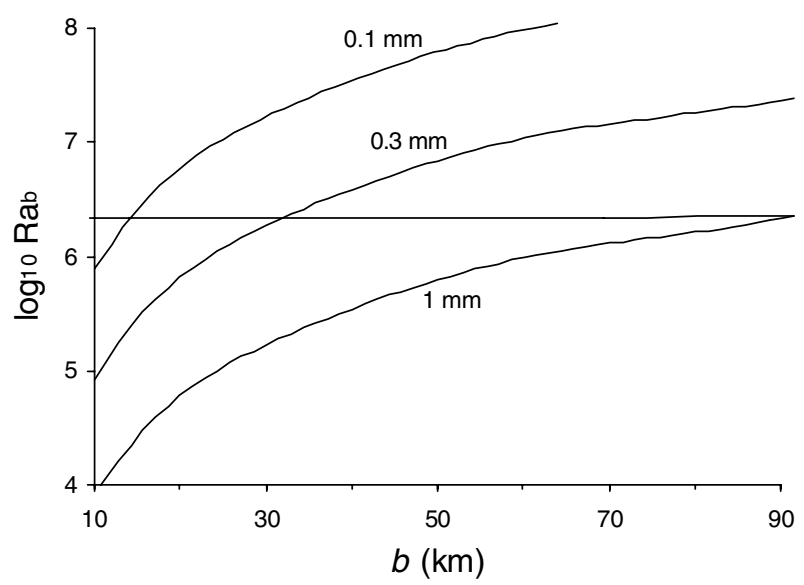

Figure 2. $R a_{\mathrm{b}}$ (labeled curves) and $R a_{\mathrm{b}} *$ (subhorizontal line) shown as a function of the thickness of a conductive ice shell for diffusion creep, $T_{\mathrm{s}}=100 \mathrm{~K}$, and grain sizes of $0.1,0.3$, $1 \mathrm{~mm}$.

solid-state greenhouse in the uppermost superficial ice layer (e.g., Matson and Brown, 1989)) reduces the ice shell thickness required for the onset of convection. However, the critical heat flow at the onset of convection is almost independent of the surface temperature (Ruiz and Tejero, 2003).

A lower limit for the heat flow through the ice shell can be taken from the current contribution due only to radioactive heat sources, $\sim 6-8 \mathrm{~mW} \mathrm{~m}^{-2}$ (Cassen et al., 1982; Squyres et al., 1983; Hussmann et al., 2002; Spohn and Schubert, 2003). For a model of Europa that includes dynamic decoupling between icy shell and rocky core by an ocean, the minimum contribution to the heat flow by tidal heating in the core would be $\sim 12-16 \mathrm{~mW} \mathrm{~m}^{-2}$ (Squyres et al., 1983; Ross and Schubert, 1987). Thus, heat flow reaching to the icy shell base from the rock and metal core would be at least $\sim 20 \mathrm{~mW} \mathrm{~m}^{-2}$ (which is equivalent to a shell at most $\sim 30 \mathrm{~km}$ thick). A certain contribution to the total surface heat flow of Europa would be provided by tidal heating within the icy shell: in a conductive ice shell this contribution would be $\sim 7 \mathrm{~mW} \mathrm{~m}^{-2}$ (Ojakangas and Stevenson, 1989). As above noted, the part of a conductive shell that contributes most to total tidal heating is the deep and warm ice near its base, which allows us to assume that tidal heat in supplied to the base (tidal heating in a convective ice shell would be greater; e.g., McKinnon, 1999; Ruiz and Tejero, 2003). Considering all heat sources, the heat flow through the ice shell would be $\sim 25-30 \mathrm{~mW} \mathrm{~m}^{-2}$ or higher (equivalently, $b \leq 19-23 \mathrm{~km}$ ).

Thus, for diffusion creep, the onset of convection could only have occurred in Europa's icy shell for grain sizes less than $\sim 0.2 \mathrm{~mm}$. The onset of convection would be marginally possible for grain sizes of $\sim 1 \mathrm{~mm}$, but only if 
non-radioactive contributions to the total heat budget are neglected (and for an ice shell $\sim 90 \mathrm{~km}$ thick), which is not satisfactory for Europa.

For superplastic flow and grain sizes in the range $0.1-1 \mathrm{~mm}$ the onset of convection requires a critical heat flow of, at most, $\sim 25-45 \mathrm{~mW} \mathrm{~m}^{-2}$, or an ice shell (for $T_{\mathrm{s}} \approx 100 \mathrm{~K}$ ) at least $\sim 10-25 \mathrm{~km}$ thick (McKinnon, 1999; Ruiz and Tejero, 2003). These values are easier to satisfy than their equivalent for diffusion creep. In comparison, for dislocation creep convection requires heat flows less than $\sim 10-15 \mathrm{~mW} \mathrm{~m}^{-2}$, or an ice shell thicker than $\sim 40-50 \mathrm{~km}$ (McKinnon, 1999; Ruiz and Tejero, 2003).

In conclusion, if the feasibility of diffusion creep for water ice is accepted (it is worth recalling that water ice flow has not observed for diffusion creep), convection in the ice shell of Europa could have initiated for this flow mechanism, although significantly lower heat flows (equivalently higher shell thicknesses) and/or grain sizes than superplastic flow are required. Accordingly, if the ice shell is convective, the most probable flow mechanism is superplastic flow. This result is consistent with those derived from laboratory experiments. Thus, superplastic flow should be considered the most likely rheology when modeling convection on Europa, prompting further analysis of non-Newtonian convection on this remarkable Galilean satellite.

\section{Acknowledgements}

The authors thank critical comments from an anonymous referee, which greatly improved this paper, and Ana Burton for linguistic assistance.

\section{References}

Barr, A. C. and Pappalardo, R. T.: 2003, 'Numerical Simulations of Non-Newtonian Convection in Ice: Application to Europa', Lunar Planet. Sci. 34th, abstract 1477.

Cassen, P. M., Peale, S. J., and Reynolds, R. T.: 1982, 'Structure and Thermal Evolution of the Galilean Satellites', in D. Morrison (ed.), Satellites of Jupiter, University of Arizona Press, Tucson, pp. 93-128.

Chizhov, V. E.: 1993, 'Thermodynamic Properties and Thermal Equation of State of HighPressure Ice Phases', Prikl. Mekh. Tekh. Fiz. (Engl. Transl.) 2, 113-123.

Durham, W. B. and Stern, L. A.: 2001, 'Rheological Properties of Water Ice - Applications to Satellites of the Outer Planets', Annu. Rev. Earth Planet. Sci. 29, 295-330.

Geissler, P. E., et al.: 1998, 'Evolution of Lineaments on Europa: Clues from Galileo Multispectral Imaging Observations', Icarus 135, 107-126.

Goldsby, D. L. and Kohlstedt, D. L.: 2001, 'Superplastic Deformation of Ice: Experimental Observations', J. Geophys. Res. 106, 11017-11030.

Hussmann, H., Spohn, T., and Wieczerkowski, K.: 2002, 'Thermal Equilibrium States of Europa's ice shell: Implications for Internal Ocean Thickness and Surface Heat Flow', Icarus 156, 143-151. 
Kirk, R. L. and Stevenson, D. J.: 1987, 'Thermal Evolution of a Differentiated Ganymede and Implications for Surface Features', Icarus 69, 91-134.

Klinger, J.: 1980, 'Influence of a Phase Transition of the Ice on the Heat and Mass Balance of Comets', Science 209, 271-272.

Matson, D. L. and Brown, R. H.:1989, 'Solid-State Greenhouses and Their Implications for Icy Satellites', Icarus 77, 67-81.

McKinnon, W. B.: 1999, 'Convective Instability in Europa's Floating Ice Shell', Geophys. Res. Lett. 26, 951-954.

Nimmo, F. and Manga, N.: 2002, 'Causes, Characteristics and Consequences of Convective Diapirism on Europa', Geophys. Res. Lett. 29, 2109, 10.1029/2002GL015754.

Ojakangas, G. W. and Stevenson, D. J.: 1989, 'Thermal State of an Ice Shell on Europa', Icarus 81, 220-241.

Pappalardo, R. T., et al.: 1998, 'Geological Evidence for Solid-State Convection in Europa's Ice Shell', Nature 391, 365-368.

Ross, M. N. and Schubert, G.: 1987, 'Tidal Heating in an Internal Ocean Model of Europa', Nature 325, 133-134.

Ruiz, J. and Tejero, R.: 2003, 'Heat Flow, Lenticulae Spacing, and Possibility of Convection in the Ice Shell of Europa', Icarus 162, 362-373.

Shoemaker, E. M., Lucchitta, B. K., Wilhelms, D. E., Plescia, J. B., and Squyres, S. W.: 1982, 'The Geology of Ganymede', in D. Morrison (ed.), Satellites of Jupiter, University of Arizona Press, Tucson, pp. 435-520.

Showman, A. P. and Han, L.: 2004, 'Numerical Simulations of Convection in Europa's Ice Shell: Implications for Surface Features', J. Geophys. Res. 109, E01010, 10.1029/ 2003JE002103.

Solomatov, V. S.: 1995, 'Scaling of Temperature- and Stress-Dependent Viscosity Convection', Phys. Fluids 7, 266-274.

Sotin, C., Head, J. W., and Tobie, G.: 2002, 'Europa: Tidal Heating of Upwelling Thermal Plumes and the Origin of Lenticulae and Chaos Melting', Geophys. Res. Lett. 29, 10.1029/ 2001 GL013844.

Spohn, T. and Schubert, G.: 2003, 'Oceans in the Icy Galilean Satellites?', Icarus 161, 456-467.

Squyres, S. W., Reynolds, R. T. Cassen, P. M., and Peale, S. J.: 1983, 'Liquid Water and Active Resurfacing on Europa', Nature 301, 225-226.

Tobie, G., Choblet, G., and Sotin, C.: 2003, 'Tidally Heated Convection: Constraints on Europa's Ice Shell Thickness', J. Geophys. Res. 108, 5124, 10.1029/2003JE002099. 